\title{
Liquid-Liquid Phase Transition in Confined Water: A Monte Carlo Study
}

\author{
Martin Meyer ${ }^{\dagger}$ and H. Eugene Stanley* \\ Center for Polymer Studies and Department of Physics, Boston University, Boston, Massachusetts 02215
}

Received: October 23, 1998; In Final Form: April 20, 1999

\begin{abstract}
We report simulations of water confined between two parallel flat (hydrophobic) walls separated by $10.95 \AA$. Our results are consistent with the possibility of a liquid-liquid phase transition separating two phases of different densities. The low-density liquid phase displays tetrahedral "ice-like" local ordering, while the highdensity liquid phase shows indications of a hexagonal layer structure similar to the quasi-2D ice forms recently found in simulations using the TIP4P potential.
\end{abstract}

Amorphous solid water displays two distinct phases, low density amorphous ice (LDA) and high density amorphous ice (HDA) that transform into each other via a first order phase transition. ${ }^{1}$ Recently, it has been proposed that the transition line between these known phases can be extrapolated to higher temperatures into the metastable liquid region of the phase diagram, raising the intriguing possibility of the coexistence of two liquid phases and of the existence of a second critical point in the metastable liquid. ${ }^{2}$ This "liquid-liquid phase transition" scenario has been supported by computer simulations with various effective interaction potentials, specifically with the ST2, ${ }^{2,3}$ TIP4P, ${ }^{2,4}$ and the SPC/E ${ }^{5}$ potentials. Also, it has been shown that simple lattice models of water ${ }^{6}$ as well as simplified spherically symmetric soft core potentials ${ }^{7}$ and mean field approaches $^{8}$ can display a liquid-liquid-phase transition. Experimental evidence for the existence of the second critical point is difficult to obtain, since the strong nucleation tendency in the region of the phase diagram, where the second critical point might be found, makes it impossible to keep water in the liquid state. $^{9}$

It is not clear whether the liquid-liquid phase transition occurs also for strongly confined water. Water in confined systems plays an important role in many biological and geological systems, ${ }^{10,11}$ and methods have been developed to experimentally test the behavior of molecularly thin water films confined between hydrophilic ${ }^{12,13}$ as well as hydrophobic ${ }^{14}$ and metal ${ }^{15}$ surfaces. In some of these systems the freezing of water seems inhibited, ${ }^{14,16}$ thus allowing one to study the phase diagram of water in regions not accessible for bulk water.

Apart from a few recent studies, ${ }^{17,18}$ computer simulations of confined water have mostly been done at ambient temperatures. ${ }^{19-22}$ Here we use the Monte Carlo method to study strongly confined supercooled ST2 water. Our results are consistent with the possibility that a liquid-liquid phase transition occurs for similar temperatures and pressures as for the bulk. Further, we find that the anisotropy of the confined system plays a key role in determining the phase behavior. In constant density simulations, phase transition behavior can be found only for the lateral pressure $P_{x y}$ (parallel to the walls), not for the transverse pressure $P_{z}$ (perpendicular to the walls).

\footnotetext{
$\dagger$ Present address: Science I\& Finance, 109-111 rue Victor Hugo, 92532 Levallois $\{\backslash$ sc cedex $\}$, France.

$\ddagger$ This manuscript was originally submitted for publication in the $\mathrm{C}$. Austen Angell Festschrift, published May 20, 1999.
}

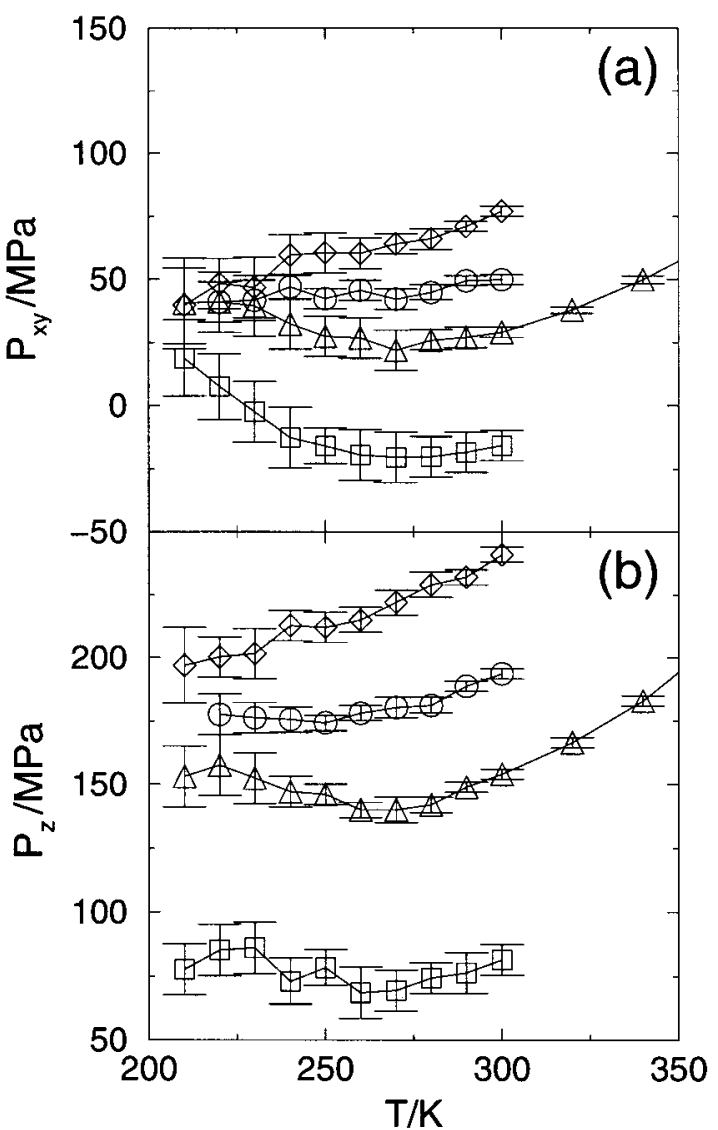

Figure 1. (a) The lateral (parallel) pressure $P_{x y}$ and (b) the transverse (perpendicular) pressure $P_{z}$ as functions of temperature for isochores with densities $\rho=0.73 \mathrm{~g} / \mathrm{cm}^{3}(\square), 0.79 \mathrm{~g} / \mathrm{cm}^{3}(\triangle), 0.82 \mathrm{~g} / \mathrm{cm}^{3}(O)$, and $0.86 \mathrm{~g} / \mathrm{cm}^{3}(\diamond)$. The densities have been calculated by using the accessible volume $V^{\prime} \equiv L_{x} L_{y}\left(L_{z}-2 z_{0}+r_{0}\right)$, where $z_{0}$ is the LennardJones radius of the wall-molecule interaction and $r_{0}$ is the LennardJones radius of the ST2 potential. The error bars have been estimated by calculating the fluctuations for eight independent configurations.

Our system consists of $N=216$ water molecules interacting with each other via the ST2 potential, ${ }^{23}$ and interacting with the walls via the 9-3-Lennard-Jones potential $\varphi(z)=\mathrm{r}\left\{\left(z_{0} /\right.\right.$ $\left.z)^{9}-\left(z_{0} / z\right)^{3}\right\}$. Here $z$ measures the distance between the center of the oxygen atom and the wall, and $c=52 \mathrm{meV}$ and $z_{0}=$ $2.47 \AA$, parameter values typically used to mimic the interaction between water and carbon. ${ }^{21}$ The distance between the walls is 


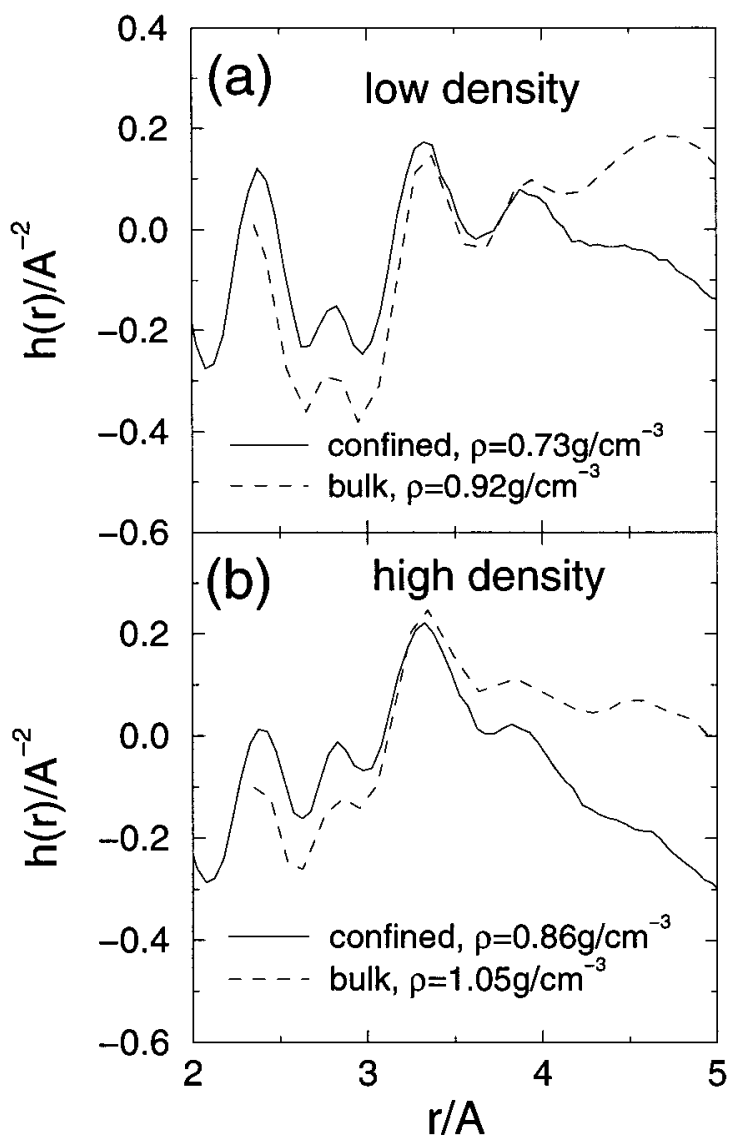

Figure 2. The neutron weighted correlation function $h(r)$ for the confined system at $T=220 \mathrm{~K}$ (full lines) in comparison to the bulk at $T=235 \mathrm{~K}$ (dashed lines) (a) in a low-density configuration and (b) in a high-density configuration. The bulk data are redrawn from ref 2 (constant volume MD simulations at $235 \mathrm{~K}$, the pressures in the lowand high-density phases are $209 \mathrm{MPa}$ and $218 \mathrm{MPa}$ ).

$L_{z}=10.95 \AA$, and periodic boundary conditions are applied in the $x y$ directions. We use a cutoff at $r=8 \AA$ for the long-range Coulomb interactions and perform constant density simulations for several values of the density $\rho$.

Because of the strong system anisotropy, $P_{x y} \neq P_{z}$, where $P_{x y} \equiv L_{z}^{-1}(\partial F / \partial A)_{\mathrm{NTL}_{z}}$ and $P_{z} \equiv A^{-1}\left(\partial F / \partial L_{z}\right)_{N T A}, F$ is the Helmholtz free energy, and $A \equiv L_{x} L_{y}$ is the area of the walls. Because of the periodic boundary conditions, finite size effects are strongly suppressed and the system can be regarded as macroscopic in the $x y$ direction. In the $z$ direction on the other hand, finite size effects are expected to be very strong. Hence the free energy is extensive with regard to $A$,

$$
F\left(N, A, L_{z}, T\right)=A f\left(N / A, L_{z}, T\right)
$$

and not with regard to $L_{z}$-i.e., it is not the case that $F\left(N, A, L_{z}\right.$, $T)=L_{z} f\left(N / L_{z}, A, T\right)$. Equation 1 shows that $P_{x y}$ and $A$ are the quantities analogous to the bulk pressure and the bulk volume. Especially, one expects the signatures of phase coexistence to be found in $P_{x y}$ and $A$ rather than in $P_{z}$ and $L_{z}$, since phase separation can occur much more easily in the $x y$ direction than in the $z$ direction (a smaller interface is required). For a constant volume simulation, phase coexistence will result in densityindependent $P_{x y}$.

Figure 1 shows (a) $P_{x y}$ and (b) $P_{z}$ as functions of temperature for different (constant) densities. We have calculated $P_{x y}$ from the $x y$ virial, and $P_{z}$ from the force on the walls. For low densities, the curves display minima, indicating the existence of a temperature of maximum density (TMD) line (where the

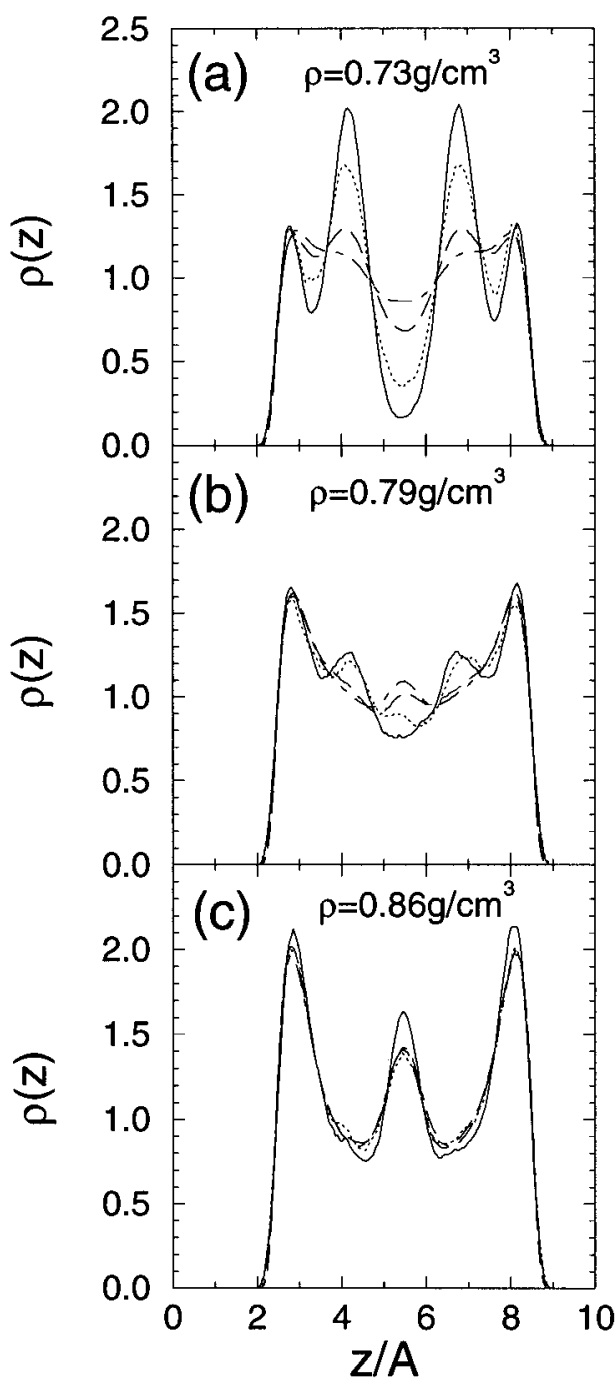

Figure 3. The $z$ dependent density $\rho(z)$ as a function of $z$ for (a) $\rho=$ $0.73 \mathrm{~g} / \mathrm{cm}^{3}$, (b) $\rho=0.79 \mathrm{~g} / \mathrm{cm}^{3}$, and (c) $\rho=0.86 \mathrm{~g} / \mathrm{cm}^{3}$. The temperatures are $T=210 \mathrm{~K}(-), 240 \mathrm{~K}(\cdots), 270 \mathrm{~K}(---)$, and $300 \mathrm{~K}$ $(-\cdot-\cdot)$.

density is a maximum as a function of temperature for constant pressure, as can be seen from the relation $(\partial P / \partial T)_{V}-(\partial P / \partial V)_{T^{-}}$ $(\partial V / \partial T)_{P}{ }^{2,10}$. The $P_{x y}$ curves for different densities become closer at lower temperatures and seem to collapse below $T=230 \mathrm{~K}$, indicating a density-independent pressure (within the error bars of the simulation), typical for the coexistence of two phases with different densities. ${ }^{2}$ Analogous behavior is not found for $P_{z}$, which remains density-dependent down to the lowest simulation temperatures. The fact that the curves do not collapse for $P_{z}$ is consistent with the results of constant $P_{z}$ simulations reported in ref 18 .

In order to compare our results with the behavior of bulk water, we calculate the neutron weighted pair correlation function, which is a weighted superposition of the $\mathrm{O}-\mathrm{O}-, \mathrm{O}-\mathrm{H}-$, and $\mathrm{H}-\mathrm{H}$-pair correlation functions

$$
h(r)=4 \pi \rho\left[0.092 g_{\mathrm{OO}}(r)+0.422 g_{\mathrm{OH}}(r)+0.486 g_{\mathrm{HH}}(r)\right]
$$

(for the coefficients in (2) we use the values for $\mathrm{D}_{2} \mathrm{O}$ to be consistent with ref 2 ). Here the pair correlation functions are calculated the same way as for a bulk system (i.e., by ignoring the anisotropy of the system).

It is known for bulk water that the function $h(r)$ shows differences for ST2 as compared to real water, although it 


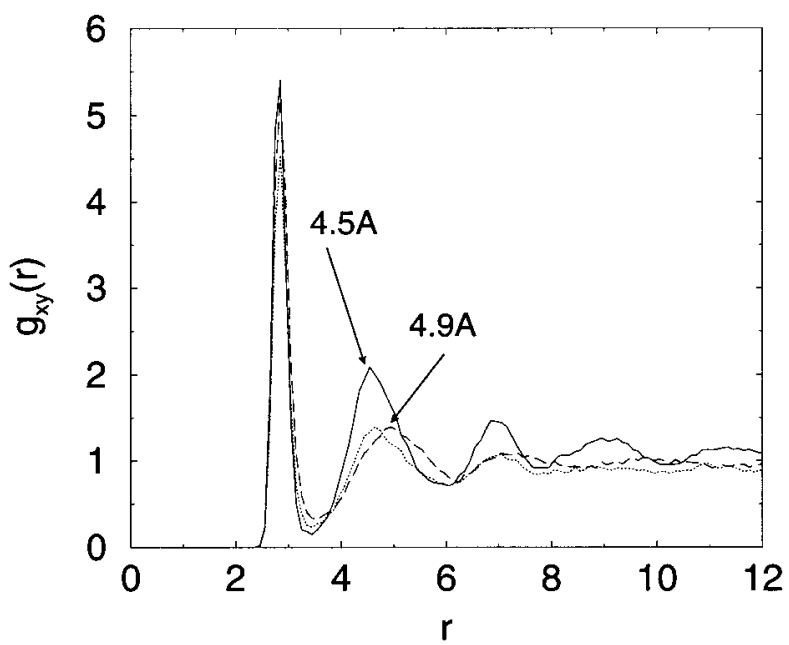

Figure 4. The lateral pair distribution function $g_{x y}(r)$, for $T=210 \mathrm{~K}$ and $\rho=0.73 \mathrm{~g} / \mathrm{cm}^{3}(-), 0.79 \mathrm{~g} / \mathrm{cm}^{3}(\cdots)$, and (c) $0.86 \mathrm{~g} / \mathrm{cm}^{3}(--)$, where $r \equiv \sqrt{x^{2}+y^{2}}$.

behaves similar in general (see, e.g., ref 2). Since our intention here is to study possible analogies between the confined and the bulk system of ST2, we compare our results with bulk ST2 water.

In Figure 2, we compare the neutron weighted pair correlation functions of two densities on the high- and low-density sides of the coexistence regime with the corresponding quantities in the bulk (taken from ref 2). Within the range $2 \AA<r<5 \AA$, the curves for the confined system are similar to the bulk, indicating that the local structures of the low- and high-density phases in the confined system are similar to their bulk analogues.

Note that the similarity between the $h(r)$ in the confined system and in the bulk makes it seem extremely unlikely that the phase transition behavior found here depends on the specific choice of the distance between the walls.

To study the differences between the high and low-density phases in more detail, we show in Figure 3 the $z$ dependent density $\rho(z)$ as a function of $z$, for different average densities $\rho$. At low densities (Figure 3a), the fluid tends to form two layers that each split into two sublayers. At high densities (Figure 3c), the fluid tends to form three flat (i.e., unsplit) layers, thereby fitting more molecules to the available volume. The occurrence of flat (as opposed to split) layers makes the high-density fluid similar to the (flat) 2D high-pressure ice phase found in simulations of confined TIP4P water by Koga et al. ${ }^{17}$

The latter finding is confirmed by the lateral $\mathrm{O}-\mathrm{O}$ pair distribution function $g_{x y}(r)$ (Figure 4), where $g_{x y}(r)$ is the number of molecules in a volume element at the same $z$ coordinate and lateral distance $r \equiv \sqrt{x^{2}=y^{2}}$ from a randomly chosen molecule, normalized by the number density of molecules. For all densities, $g_{x y}(r)$ decays very fast to a constant, thereby confirming that the system does not show crystallization. The location of the first maximum at about $2.8 \AA$ does not change much when increasing the density. In contrast, we find that the second maximum is shifted from about $4.5 \AA$ to approximately $4.9 \AA$. We interpret that shift as a change of the bond angle inside the layers from approximately $110^{\circ}$ to $120^{\circ}$. This finding is consistent with a change from a tetrahedral local structure at low densities to layers with approximately honeycomb structure similar to the crystal structures found in ref 17.

Our results suggest that the liquid-liquid pase transition occurs in strongly confined water modeled by the ST2 potential. The neutron weighted radial distribution functions of the two liquids in the confined system resemble their analogues in the bulk. The more detailed analysis in Figures 3 and 4, which takes into account the system anisotropy, reveals similarities between the high-pressure liquid and the flat ice phases ${ }^{17}$ found recently in confined systems.

Acknowledgment. We thank M.-C. Bellissent-Funel, M. Canpolat, R. Sadr-Lahijany, S. Sastry, A. Scala, F. W. Starr, and D. Wolf for very helpful discussions, S. Harrington for very valuable collaboration in the early stages of this research, and DFG and NSF for financial support.

\section{References and Notes}

(1) Mishima, O.; Calvert, L. D.; Whalley, E. Nature 1985, 314, 74. Mishima, O. J. Chem. Phys. 1994, 100, 5910.

(2) Poole, P. H.; Sciortino, F.; Essmann, U.; Stanley, H. E. Nature 1992, 360, 324; Phys. Rev. E 1993, 48, 3799.

(3) Harrington, S. T.; Zhang, R.; Poole, P. H.; Sciortino, F.; Stanley, H. E. Phys. Rev. Lett. 1997, 78, 2409.

(4) Tanaka, H. Nature 1996, 380, 328; J. Chem. Phys. 1996, 105, 5099. (5) Harrington, S.; Poole, P. H.; Sciortino, F.; Stanley, H. E. J. Chem Phys. 1997, 107, 7443.

(6) Borick, S. S.; Debenedetti, P. G.; Sastry, S. J. Chem. Phys. 1995, 99, 3781. See also, Sastry, S.; Debenedetti, P. G.; Sciortino, F.; Stanley, H. E. Phys. Rev. E 1996, 53, 6144.

(7) Sadr-Lahijany, M. R.; Scala, A.; Buldyrev, S. V.; Stanley, H. E., cond-mat/9806091. Hemmer, P. C.; Stell, G. Phys. Rev. Lett. 1970, 24 , 1284.

(8) Poole, P. H.; Sciortino, F.; Grande, T.; Stanley, H. E.; Angell, C. A. Phys. Rev. Lett. 1994, 73, 1632.

(9) Recent evidence has been obtained by indirect methods. See, e.g., Bellissent-Funel, M.-C. Europhys. Lett. 1998, 42, 161. Mishima, O.; Stanley, H. E. Nature 1998, 392, 164. Debenedetti, P. G. Nature 1998, 392, 127.

(10) Debenedetti, P. G. Metastable Liquids: Concepts and Principles; Princeton University Press: Princeton, 1996.

(11) Zheng, Q.; Durben, D. J.; Wolf, G. H.; Angell, C. A. Science 1991, 254,829 .

(12) Dore, J. In Correlations and Connectivity: Geometric Aspects of Physics, Chemistry, and Biology; Stanley, H. E., Ostrowsky, N., Eds.; Kluwer: Dordrecht, 1990.

(13) Bellissent-Funel, M. C.; Chen, S. H.; Zanotti, J. M. Phys. Rev. E 1995, 51,4558

(14) Bellissent-Funel, M.-C.; Sridi-Dorbez, R.; Bosio, L. J. Chem. Phys. 1996, 104, 10023

(15) Porter, J. D.; Zinn-Warner, A. S. Phys. Rev. Lett. 1994, 73, 2879. 1478.

(17) Koga, K.; Zeng, X. C.; Tanaka, H. Phys. Rev. Lett. 1997, 79, 5262.

(18) Koga, K.; Zeng, X. C.; Tanaka, H. Chem. Phys. Lett. 1998, 285 , 278.

(19) Wallquist, A. Chem. Phys. Lett. 1990, 165, 437.

(20) Zhu, S. B.; Robinson, G. W. J. Chem. Phys. 1990, 94, 1403.

(21) Lee, S. H.; Rossky, P. J. J. Chem. Phys. 1994, 100, 3334.

(22) Hartnig, C.; Witschel, W.; Spohr, E. J. Phys. Chem. 1998, 102, 1241.

(23) Stillinger F. H.; Rahman, A. J. Chem. Phys. 1974, 60, 1545. 